\title{
ARTICLE
}

\section{Magnesium lithospermate B ameliorates microcirculation perfusion in rats by promoting vascular NO production via activating the PI3K/AKT pathway}

\author{
Ying-luo Liu ${ }^{1,2}$, Xiao-yu Zhou ${ }^{1}$ and Li-jiang Xuan ${ }^{2,3}$
}

\begin{abstract}
Microcirculation morphologically refers to the blood flow in vessels of less than $150 \mu \mathrm{m}$ in diameter, including arterioles, capillaries and venules, which provides nutrients and removes metabolic byproducts within tissues. Microcirculation dysfunction is involved in the pathological progress of many diseases, such as obesity, hypertension, and insulin resistance. In this study we investigated the effects of magnesium lithospermate B (MLB), an active compound of the traditional Chinese medicine Slavia miltiorrhiza, on the microcirculation dysfunction in rats and the underlying molecular mechanisms. The effects of MLB on microcirculation were assessed in vivo by measuring the hindlimb blood perfusion in dextran-induced microcirculation dysfunction rats and mesentery blood flow in anesthetized rats. We demonstrated that administration of MLB restored the impaired rat hindlimb blood flow and promoted the mesenteric micoperfusion in vivo. We further revealed in these two animal models that MLB treatment significantly increased the production of total nitrite in vascular tissues (mesentery, aorta, and heart), which was confirmed in human microvascular endothelial cells (HMEC-1) treated with MLB in vitro. Moreover, we showed that MLB treatment significantly increased the phosphorylation of endothelium nitric oxide synthase (eNOS) via inducing AKT phosphorylation in vivo and in vitro. Co-administration of the eNOS inhibitor L-NAME $(20 \mathrm{mg} / \mathrm{kg})$ abolished the protective effects of MLB against dextran-induced microcirculation dysfunction in rats, whereas pretreatment with PI3K inhibitor LY294002 (10 $\mu \mathrm{M})$ prevented eNOS activation in MLBtreated HMEC-1 cells. Our results suggest that MLB can restore the microcirculation dysfunction via activating eNOS, and in turn enhancing the vascular nitric oxide production, which is medicated by MLB-caused activation of the PI3K/AKT pathway.
\end{abstract}

Keywords: traditional Chinese medicine; Salvia miltiorrhiza; magnesium lithospermate B; microcirculation dysfunction; NO; endothelium nitric oxide synthase; PI3K/AKT pathway

Acta Pharmacologica Sinica (2019) 40:1010-1018; https://doi.org/10.1038/s41401-018-0203-7

\section{INTRODUCTION}

Microcirculation is morphologically defined as microcirculation in vessels less than $150 \mu \mathrm{m}$ in diameter and is the smallest level of circulation in the body, occurring in arterioles, capillaries, and venules [1]. A critical function of microcirculation is to provide nutrients to and remove metabolic byproducts within tissues in response to variations in nutrient demand [2]. Microcirculation dysfunction refers to various conditions, ranging from decreased organ maximal perfusion to impaired endothelium-dependent dilation [3]. The loss of capillaries in remote organs and maldistribution of oxygen delivery represent two fundamental conditions of microcirculation dysfunction.

In recent decades, a number of studies have revealed the contribution of microcirculation dysfunction to the pathological progress of many diseases, such as obesity, hypertension, and insulin resistance [4]. For example, hypertension involves the anatomic alterations in the structure of microvessels [5]. Recently, clinical studies have reported that abnormalities in the function and structure of coronary microcirculation may occur in patients who do not present with obstructive atherosclerosis [6]. However, the risk factors of cardiovascular disease in patients without obstructive atherosclerosis are almost the same as those in patients with obstructive atherosclerosis [7]. It is believed that microvascular dysfunction is a powerful predictor of cardiovascular events, and the reversal of microvascular dysfunction may provide new approaches for the treatment and prevention of cardiovascular diseases.

Slavia miltiorrhiza (SM), also called Danshen, is a well-known component of Traditional Chinese Medicine, which is commonly used for the treatment of hyperviscosity syndrome or blood stasis [8]. Magnesium lithospermate B (MLB) is the major water-soluble bioactive component of SM. Recent studies using both in vivo and in vitro models have demonstrated that MLB shows the potential to prevent and treat cardiovascular damage [9]. Indeed, MLB appears to exhibit a broad spectrum of pharmacological activities, such as improving metabolic changes in metabolic syndrome [10], and it exhibited anti-ischemic neuroprotection in gerbils that were subjected to focal ischemia and reperfusion [11]. To date, the

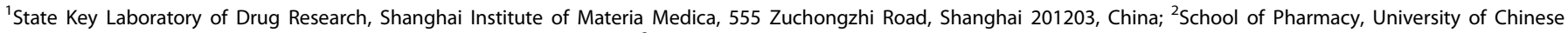

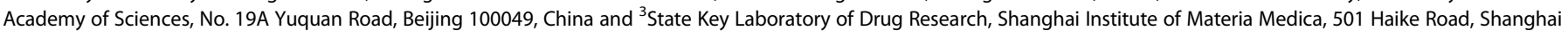
201203, China

Correspondence: Li-jiang Xuan (ljxuan@simm.ac.cn)

Received: 3 July 2018 Accepted: 4 December 2018

Published online: 13 February 2019 
molecular mechanisms of MLB in conferring these pharmacological activities remain unclear. Previous studies suggest its role in decreasing the level of ET-1 in animal models of subarachnoid haemorrhage [12] and in inhibiting lipid peroxidation in endothelial cells, thus scavenging superoxide anions and hydroxyl radicals $[13,14]$.

Our previous studies indicated that MLB attenuated myocardial ischemia-reperfusion injury in Sprague-Dawley (SD) rats [15]. It remains unclear whether MLB can improve microcirculation dysfunction. In the present study, we aim to evaluate the protective effect of MLB on microcirculation dysfunction and to explore the mechanisms behind this effect, which may lead to new therapeutic implications of MLB.

\section{MATERIALS AND METHODS}

Chemicals and reagents

Magnesium lithospermate B (purity $>98 \%$, Fig. 1) was produced in-house. The PI3K inhibitor LY294002 was obtained from Beyotime Institute of Biotechnology (Shanghai, China), and the eNOS inhibitor $N$ '-nitro- $L$-arginine-methyl ester hydrochloride $(L$ NAME) was purchased from the Dalian Meilun Biotechnology (Dalian, China). Dextran ( $M_{\mathrm{r}}$. 500000) was purchased from Bailingwei Tech (Beijing, China). Anti-eNOS and anti-phosphoeNOS (Ser1177) were obtained from Abcam (Cambridge, UK). AntiErk, anti-phospho-Erk (T202/Y204), anti-GAPDH, anti-AKT, and antiphospho-AKT (Ser473) were purchased from Cell Signaling Technology (Beverly, MA). Anti- $\beta$-actin was obtained from Sigma-Aldrich (St. Louis, MO, USA)

\section{Cell lines}

Human microvascular endothelial cells (HMEC-1) were maintained in MCDB131 medium (Gibco, CA, USA) supplemented with fetal bovine serum (FBS, Gibco), L-glutamine (Gibco), and $100 \mathrm{ng} / \mathrm{mL}$ human epidermal growth factor (Peprotech, London, UK).

\section{Quantitative RT-PCR}

The total RNA was extracted using TRIzol Reagent (Thermo Fisher Scientific, Waltham, USA) and reverse-transcribed to cDNA (Takara, Shiga, Japan). The mRNA expression of eNOS was measured by real-time PCR using specific primers and SYBR green PCR Master Mix (Bio-Rad, Hercules, CA, USA). The primer sequences of eNOS and GAPDH were as follows: eNOS (forward: 5'GTCTTCGTGGTAGCGTTGCT-3' and reverse: 5'-CCTGACAACCCAAG ACCTA-3') and GAPDH (forward: 5'-AAGAAGGTGGTGAAGCAGG-3' and reverse: 5'-AGGTGGAGGAGTGGGTGTCG-3').

\section{Cell viability assay (CCK-8 assay)}

Cell viability was assessed using CCK-8 assay (Beyotime Institute of Biotechnology, Shanghai, China). Briefly, HMEC- 1 cells $\left(10^{5}\right.$ cells/ $\mathrm{mL}$ ) were seeded into a 96-well plate and treated with MLB at doses of $50,100,200,400$, or $800 \mu \mathrm{M}$ for $2 \mathrm{~h}$ at $37^{\circ} \mathrm{C}$. The control group was treated with same volume of PBS. After treatment, 10 $\mu \mathrm{L}$ of the CCK-8 solution was added to each well and incubated at $37^{\circ} \mathrm{C}$ for $1 \mathrm{~h}$. The OD value was read at a wavelength of $450 \mathrm{~nm}$ on a SpectraMax M5 microplate reader (Molecular Devices, Sunnyvale, CA, USA) and the experiment was independently repeated three times.

Measurement of cellular NO production

NO was measured using a DAF-FM diacetate probe (Thermo Fisher, Carlsbad, USA). A total of $5 \times 10^{4}$ HMEC- 1 cells were preincubated in 96-well plates with the diluted DAF-FM diacetate for $30 \mathrm{~min}$ at $37^{\circ} \mathrm{C}$. The excess probe was removed, and the cells were treated with MLB for the indicated amount of time. Fluorescence with excitation and emission maxima at 495 and $515 \mathrm{~nm}$, respectively, was measured on a microplate reader.<smiles></smiles>

Fig. 1 The structure of magnesium lithospermate B

Western blot analyses

The total protein was lysed in RIPA buffer (Beyotime Institute of Biotechnology, China) with a protease inhibitor cocktail (Cell Signaling Technology Inc., Beverly, MA, USA). Protein $(30 \mu \mathrm{g})$ was separated using sodium dodecyl sulfate-polyacrylamide gel electrophoresis (SDS-PAGE), transferred onto a polyvinylidene difluoride (PVDF) membrane (Bio-Rad, Hercules, CA, USA), and then blocked with $5 \%$ milk at room temperature. The membranes were incubated with specific antibodies (anti-Erk 1:1000, antiphospho-Erk 1:1000, anti-eNOS 1:1000, anti-phospho-eNOS 1:1000, anti-AKT 1:1000, anti-phospho-AKT 1:1000, anti- $\beta$-actin 1:2000, and anti-GAPDH 1:2000) overnight at $4{ }^{\circ} \mathrm{C}$, followed by incubation with their corresponding secondary antibodies. The membranes were visualized by an enhanced chemiluminescence (ECL) system (Bio-Rad, Hercules, CA, USA).

In vivo experiment

Male SD rats weighing 200-250 g were obtained from the Animal Center of Shanghai Institute of Materia Medica (Shanghai, China). All of the animal studies were approved by the Animal Care and Use Committee of the Shanghai Institute of Materia Medica. The rats were housed four per cage, with the temperature maintained at $23-25^{\circ} \mathrm{C}$ under a $12 \mathrm{~h}$ light-dark cycle. All of the rats were fasted overnight before their use in the study.

Dextran-induced microcirculation disturbance evaluation: Animals were randomly divided into 2 groups ( $n=8$ per group): saline and MLB $50 \mathrm{mg} / \mathrm{kg}$. The SD rats were fixed on a thermostatcontrolled operation table (Harvard Apparatus, South Natick, USA) to maintain their body temperatures at $37^{\circ} \mathrm{C}$, stabilized for $15 \mathrm{~min}$, and then injected with $8 \%$ high molecular weight dextran $(\mathrm{v} / \mathrm{v})$ at an injection volume of $5 \mathrm{~mL} / \mathrm{kg}$ into the tail vein to induce microcirculation dysfunction. Twenty minutes later, the animals were subcutaneously dosed with MLB or the vehicle and were continuously monitored for $1 \mathrm{~h}$. A laser Doppler Perfusion Imaging System (Moor Instruments, Wilmington, DE) was used to measure hindlimb blood perfusion in rats that were anesthetized by continuous inhalation of gaseous isoflurane. For treatment with the eNOS inhibitor $L-N A M E$, animals were randomly divided into 3 groups ( $n=5$ per group), including MLB $50 \mathrm{mg} / \mathrm{kg}, L$-NAME 20 $\mathrm{mg} / \mathrm{kg}$ and $L$-NAME $20 \mathrm{mg} / \mathrm{kg}+$ MLB $50 \mathrm{mg} / \mathrm{kg}$. L-NAME $20 \mathrm{mg} / \mathrm{kg}$ was co-injected with dextran.

Determination of mesentery blood flow: Animals were randomly divided into 2 groups ( $n=10$ per group): the saline group and the MLB $50 \mathrm{mg} / \mathrm{kg}$ group. To access mesentery microcirculation, sodium pentobarbital-anesthetized rats were fixed and then the surrounding mesenteric vessels were fully exposed. The laser Doppler Needle Probe (AD instruments, Milford, MA) was placed on the exposed mesenteric vessels, which were stabilized for $15 \mathrm{~min}$ and then intravenously administered MLB or saline, after which they were continuously monitored for $30 \mathrm{~min}$. The visualization of the limb microperfusion was performed by the Powerlab recording system (AD 
instruments, Milford, MA). The laser Doppler flowmetry signal was simultaneously recorded in arbitrary blood perfusion units. The LDF ratio $=$ (maximum perfusion-baseline perfusion $) \times$ $100 \% /$ baseline perfusion.

Protein isolation and Western blot analysis: After a subcutaneous treatment with a $50 \mathrm{mg} / \mathrm{kg}$ dose of MLB or the same volume of saline, the rats were anesthetized with an intraperitoneal (ip) administration of $50 \mathrm{mg} / \mathrm{kg}$ sodium pentobarbital. The midline of the abdomen was incised and the thorax was opened to expose the heart and lung. The abdominal and thoracic aorta were cut out, and the connecting tissues were removed in cold PBS buffer. A loop of intestine from each animal was excised with surgical forceps, and the researcher rapidly removed the fat or connective tissue and then cut the vessels off. The hearts, aorta, and mesenteric vessels of rats from different treatment groups were collected and stored at $-80^{\circ} \mathrm{C}$. The aorta, mesentery, and heart tissues were homogenized on liquid nitrogen and lysed in a RIPA lysis buffer, as described previously.

Total nitrite assay: Total nitric oxide was extracted from the tissue samples with the specific nitrite lysis buffer (Beyotime Institute of Biotechnology, Shanghai, China). The concentration of nitrite/nitrate was then measured by the Griess reaction method according to the manufacturer's instructions (Beyotime Institute of Biotechnology, Shanghai, China).

Statistical analysis

Each assay was performed at least three times, and the results are expressed as the mean \pm SD. GraphPad software (San Diego, CA,
USA) was used to analyze significant differences. A $P<0.05$ was considered significant with a one-way ANOVA or the Student's $t$ test.

\section{RESULTS}

MLB restores hindlimb blood perfusion in rats with dextraninduced microcirculation dysfunction

To determine the impact of MLB on microcirculation dysfunction, we first used a dextran-induced microcirculation disturbance rat model [16]. In this study, MLB was given subcutaneously to test the potential of MLB for its sustained efficacy in restoring blood perfusion. Morphological observations noted an increased prevalence of soft tissue swelling (Fig. 2d) as well as the significantly decreased blood perfusion upon dextran treatment (Fig. 2c), suggesting a typical phenotype of microcirculation disturbance. A subcutaneous injection of MLB resulted in a notably improved hindlimb blood perfusion, as indicated by the significant increase of the LDPI ratio in the MLB-treated group compared with that of the vehicle-treated group $(P<0.05)$. These results suggested that MLB exhibited a therapeutic effect on microcirculation dysfunction.

MLB increases mesenteric microperfusion in rats

We further examined the impact of MLB on mesenteric microperfusion to evaluate its role in modulating mesenteric microcirculation. To this end, mesentery blood flow was measured in anesthetized rats that were treated with MLB or the vehicle control, and the average values of perfusion in each group are
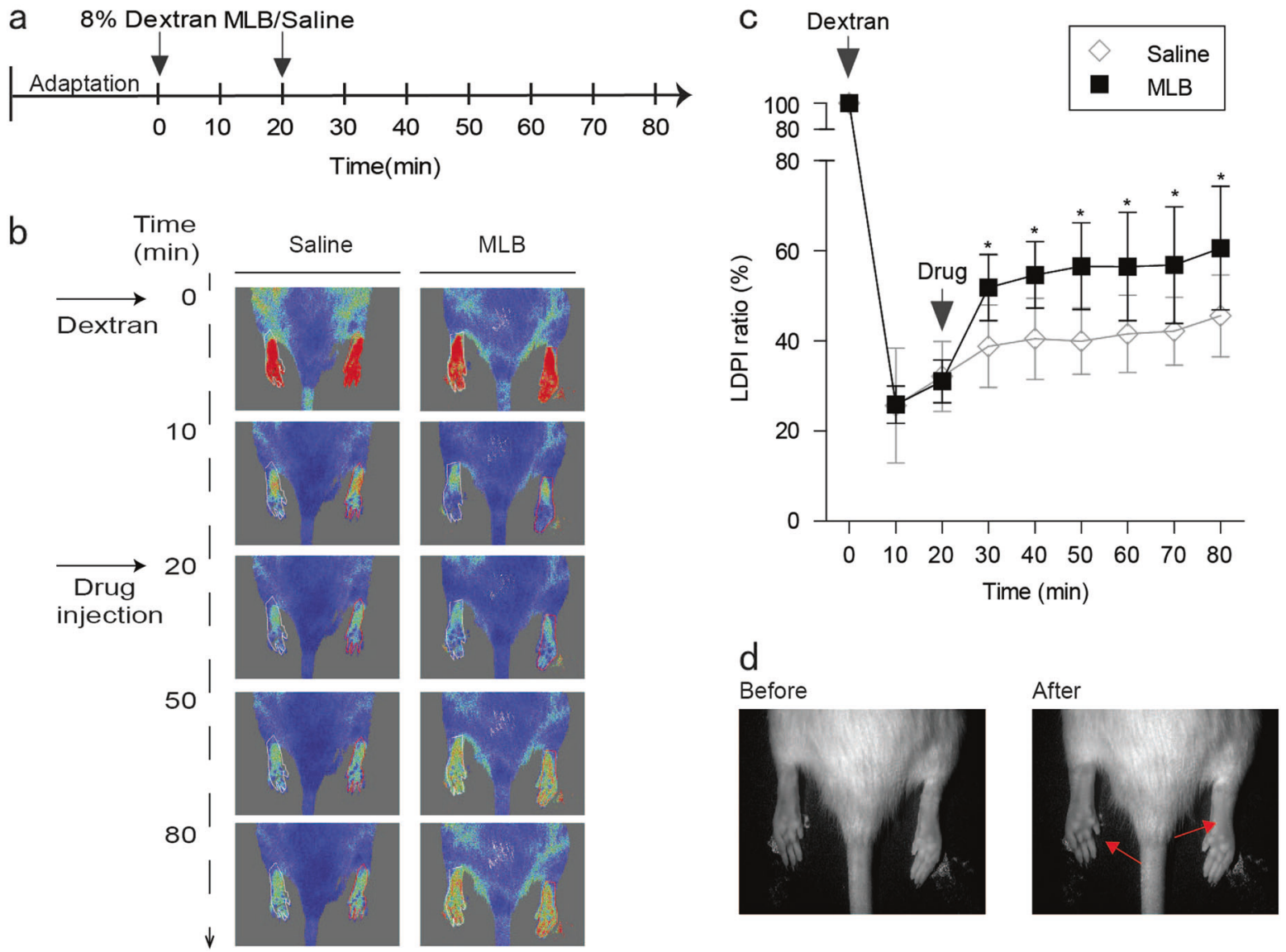

Fig. 2 MLB improved blood perfusion in rats with dextran-induced microcirculation dysfunction. a Experimental procedure to determine hindlimb perfusion, which was measured by laser Doppler perfusion imaging. b Representative LDP images of the hindlimbs. c Quantitative analysis of the blood perfusion recovery that was measured by LDPI. Ratios were calculated as the ratio of ischemic to baseline blood perfusion. Data are expressed as the mean $\pm S D, P<0.05$ vs. the vehicle group. $\mathbf{d}$ Representative photographs of rat hindlimbs before and after the dextran injection. Red arrows indicate soft-tissue swelling 
shown in Fig. 3b. We noticed that mesenteric microperfusion was dose-dependently increased by $12 \% \pm 4 \%(P<0.05), 24 \% \pm 5 \%(P$ $<0.001)$ and $37 \% \pm 5 \%(P<0.001)$ upon the administration of 12.5 $\mathrm{mg} / \mathrm{kg}, 25 \mathrm{mg} / \mathrm{kg}$ and $50 \mathrm{mg} / \mathrm{kg}$ of MLB, respectively (Fig. 3c). Arbitrary blood perfusion units were measured by the LDF (Fig. 3b), and the LDF ratio was obtained by normalization to the baseline (Fig. 3c). MLB dose-dependently increased the LDF ratio, confirming its role in ameliorating microcirculation.

MLB promotes nitric oxide production in vascular tissues We next tried to understand how MLB restored microcirculation. Increasing evidence has shown that nitric oxide (NO) [17], a principal mediator in vascular tone, could promote local relaxation of vessels and increase blood perfusion. Hence, we measured the concentrations of nitrite in vascular tissues, which were reported to be a storage pool for NO in circulation. The vessels of the representative rat vascular tissues, including the mesentery, aorta and heart, were isolated, and the concentrations of nitrite in these tissues were measured using a fluorometric nitric oxide assay. We found that treatment with MLB resulted in a significant increase in the nitrite levels in rat aorta and mesentery vessels, whereas its effect on heart vessels was marginal (Fig. 4a).

The aforementioned results suggest the increased NO production in vascular tissues. We further validated these findings in
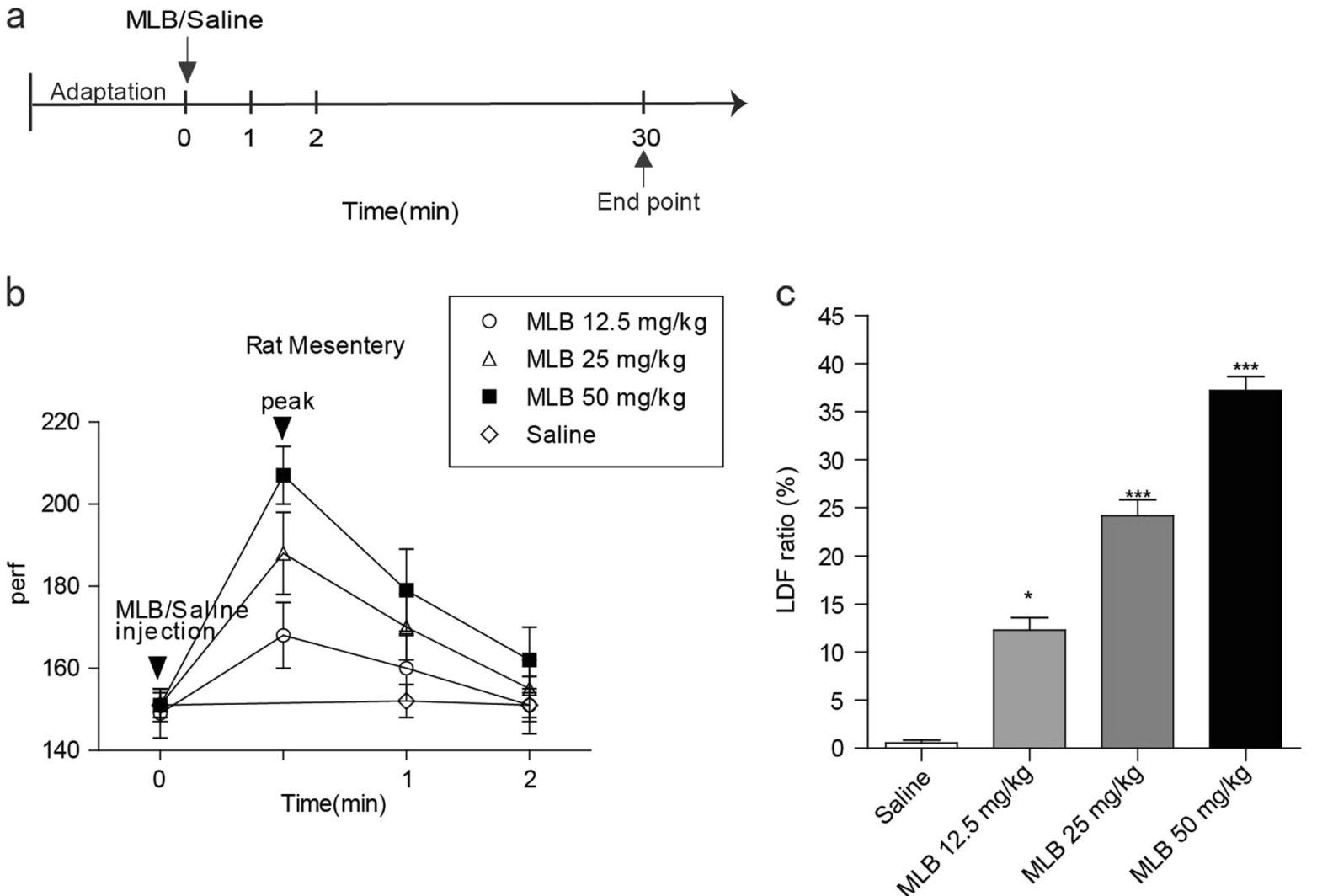

Fig. 3 MLB increased microperfusion in the rat mesentery. a Experimental procedure to determine mesentery microperfusion by laser Doppler flowmetry (LDF) ( $n=10$ per group). b Arbitrary blood perfusion units. c Quantification of the LDF ratio. The LDF ratio was obtained as described in the Materials and Methods section. Data are expressed as the mean \pm SD. ${ }^{*} P<0.05,{ }^{* *} P<0.01,{ }^{* * *} P<0.001$ vs. saline group
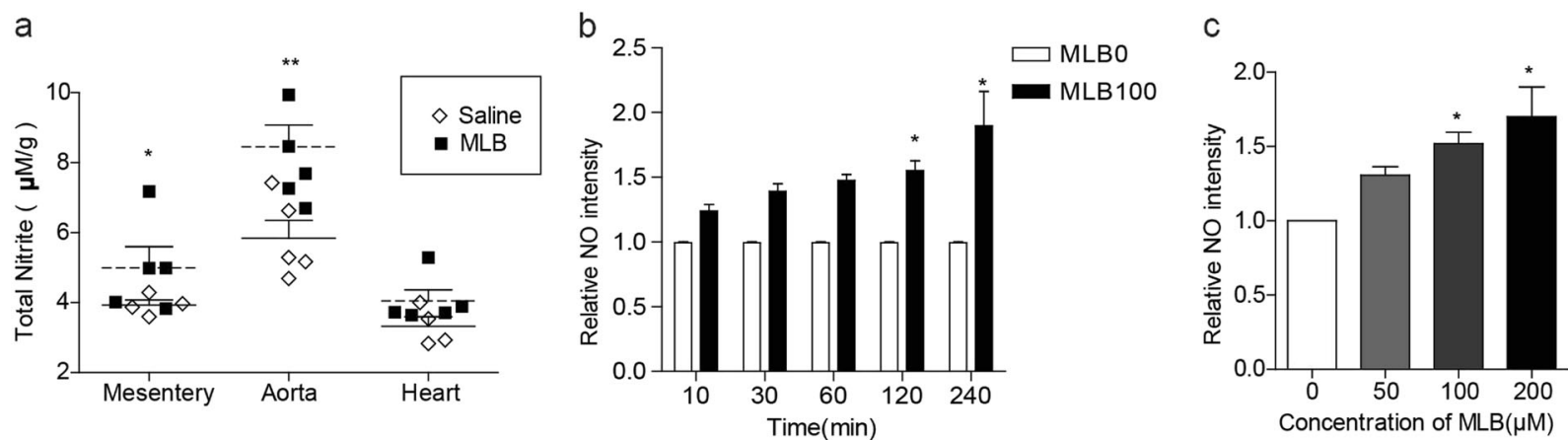

Fig. 4 MLB treatment elevated the total nitrite level. a Total nitrite level in vascular tissues. SD rats were treated with $50 \mathrm{mg} / \mathrm{kg} \mathrm{MLB}$ for $4 \mathrm{~h}$, anesthetized and the mesentery vessels, aorta, and hearts were isolated for the measurement of the total nitrite level ( $n=4-5$ each group). $\mathbf{b}$, c Relative NO intensity in HMEC-1 cells. HMEC-1 cells were preincubated with the DAF-FM diacetate probe for 30 min at $37^{\circ} \mathrm{C}$ in darkness. The excess probe was removed before treatment with MLB $(100 \mu \mathrm{M})$ for indicated time $\mathbf{b}$ or indicated dose for $2 \mathrm{~h} \mathbf{c}$. NO production was visualized by a microplate reader. Relative NO intensity was calculated by normalization to that in untreated group. ${ }^{*} P<0.05$, ${ }^{* *} P<0.01$ vs the vehicle group 

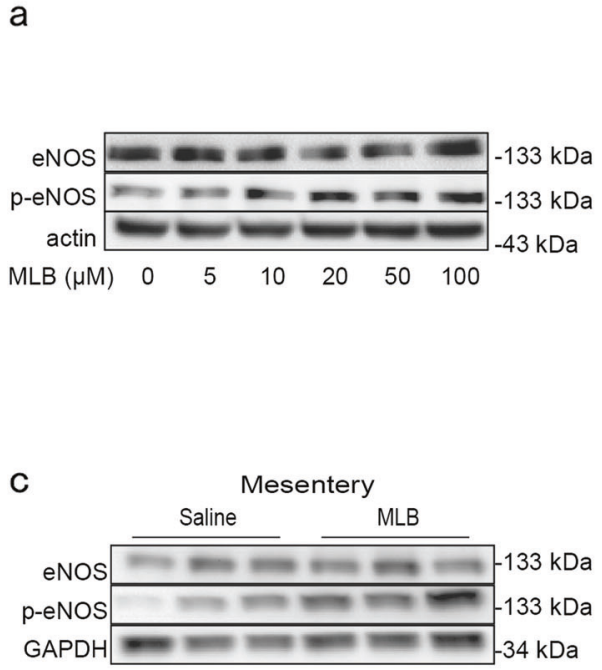
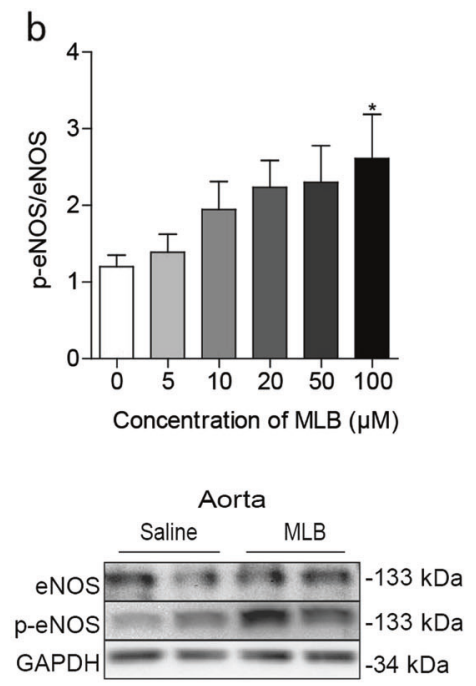
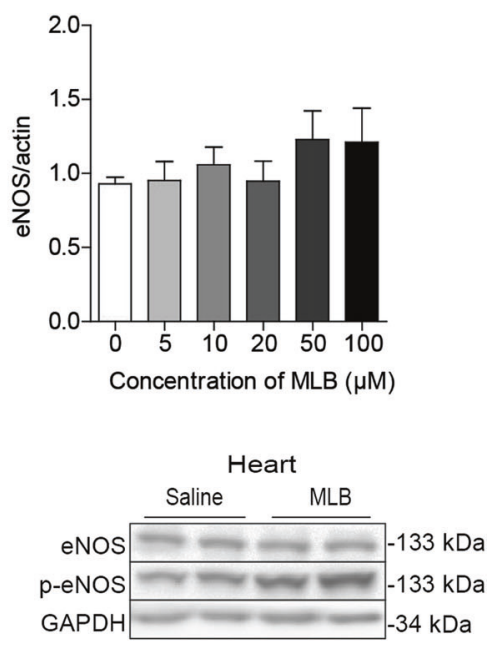
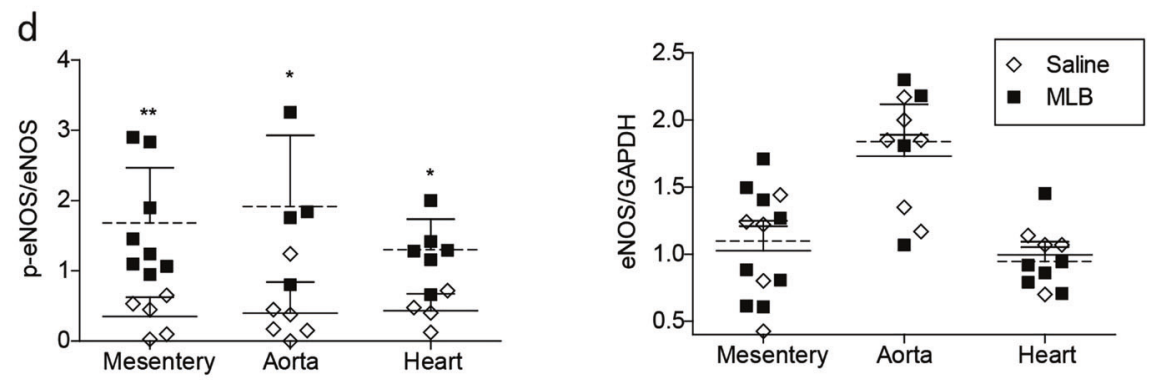

$\mathrm{e}$
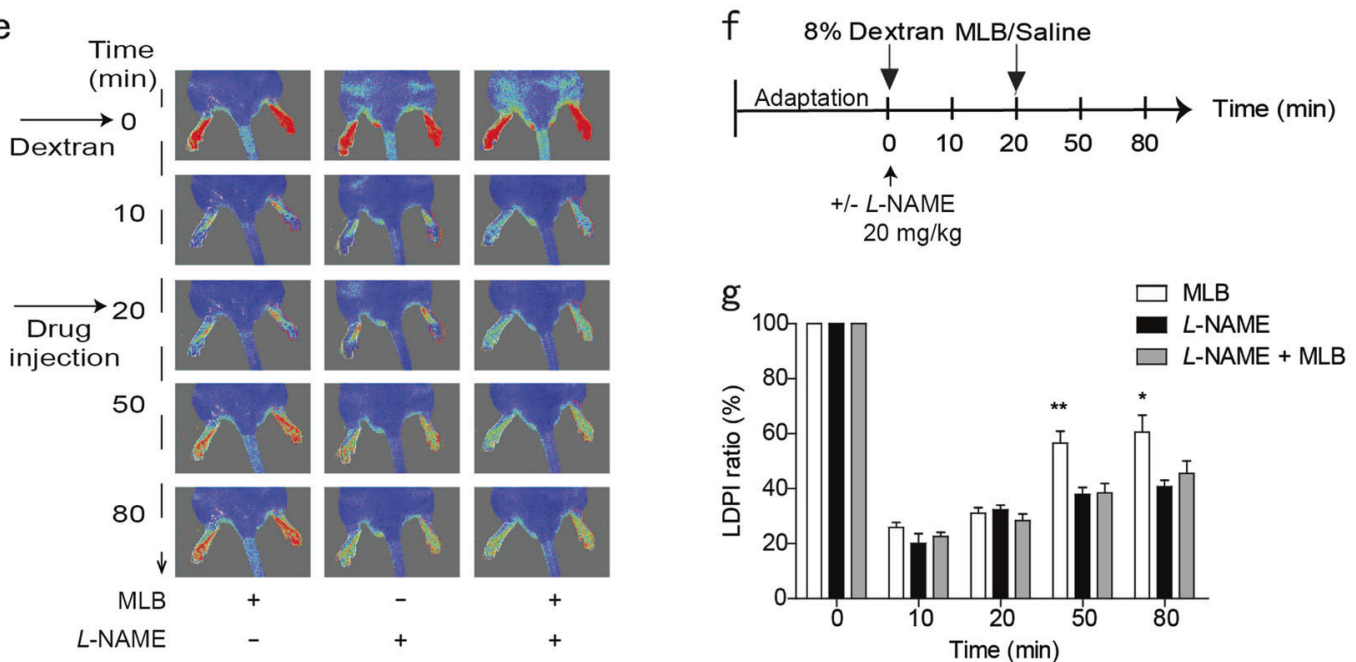

Fig. 5 MLB enhanced the phosphorylation of eNOS at Ser1177. a Representative Western blot of HMEC-1 cells. HMEC-1 were treated with different concentrations of MLB for $2 \mathrm{~h}$, followed by the Western blot analyses. $\mathbf{b}$ Quantification of eNOS expression and the p-eNOS/eNOS ratio in $\mathbf{a},{ }^{*} P<0.05$ vs. the vehicle group. c Representative Western blot in mesentery, aorta, and heart. SD rats that were treated with $50 \mathrm{mg} / \mathrm{kg}$ MLB for $4 \mathrm{~h}$ were anesthetized and the mesentery vessels, aorta, and hearts were isolated for Western blot analyses ( $n=4-8$ per group). $\mathbf{d}$ Quantification of eNOS expression and the p-eNOS/eNOS ratio in $c_{1}^{*} P<0.05,{ }^{*} P<0.01$ vs. the vehicle group. e Representative LDP images of the hindlimbs. f Experimental procedure to determine hindlimb perfusion measured by laser Doppler perfusion imaging. $\mathbf{g}$ Quantitative analysis of the blood perfusion recovery that was measured by LDPI. Ratios were calculated as the ratio of ischemic to baseline blood perfusion. Data are expressed as the mean $\pm \mathrm{SD}$. ${ }^{*} P<0.05,{ }^{* *} P<0.01 \mathrm{MLB}$ group vs. the MLB $+L-\mathrm{NAME}$ group

HMEC-1 cells that were immortalized and retained their microvascular endothelial cell characteristics. Cells were treated with non-toxic doses of MLB, as confirmed by a cell viability assay (Supplementary Fig. S1a), and the intracellular NO production was measured using the cell-permeable fluorescent probe DAF-FM. Treatment with MLB rapidly increased NO production within 120 min (Fig. 4b) in a dose-dependent manner (Fig. 4c).
MLB stimulates the phosphorylation of endothelial nitric oxide synthase

We then addressed how MLB promoted NO production. In the endothelium, NO is synthesized from $L$-arginine by endothelial nitric oxide synthase (eNOS). We hence tested whether MLB treatment could increase the expression of eNOS. MLB treatment in HMEC-1 cells barely affected eNOS at the mRNA level 
a

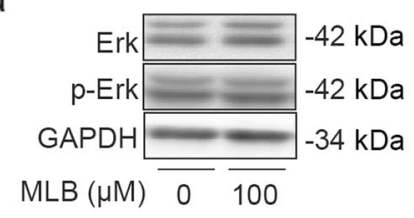

C

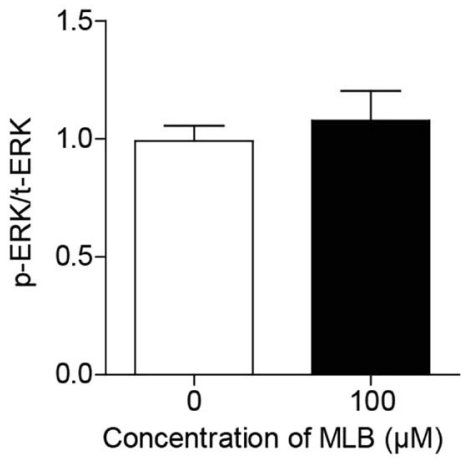

e
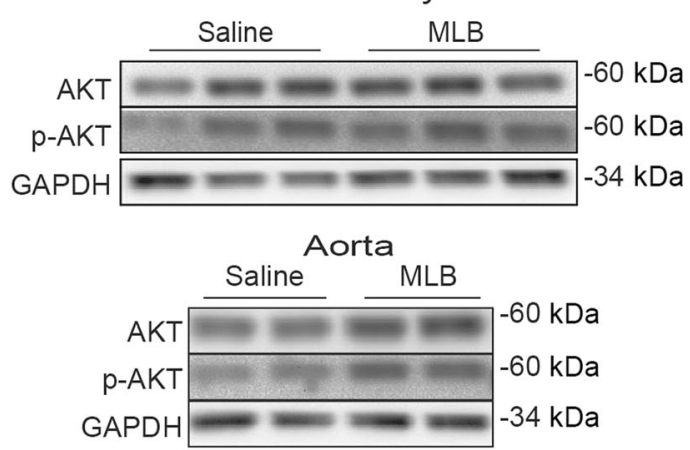

Heart

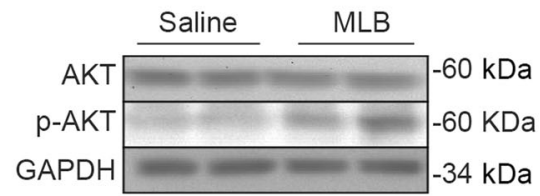

b

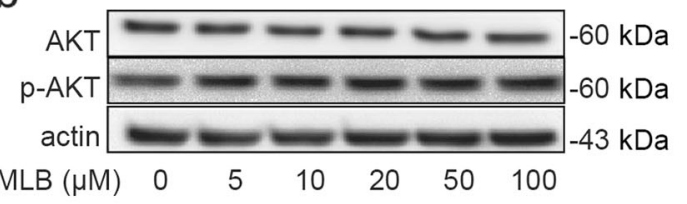

d

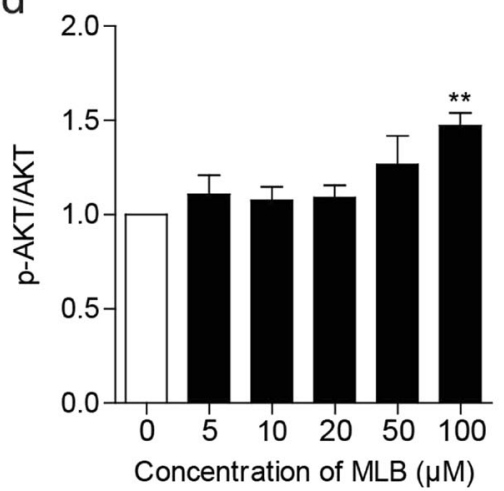

f

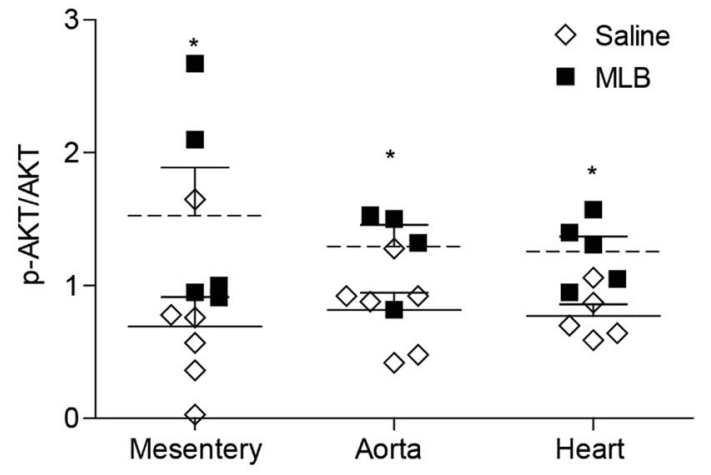

Fig. 6 MLB activated AKT phosphorylation at Ser473. $\mathbf{a}$ and $\mathbf{b}$ Representative Western blots in HMEC-1 cells. Cells were treated with different concentrations of MLB for $2 \mathrm{~h}$ followed by the Western blot analyses of the indicated proteins. $\mathbf{c}$ and $\mathbf{d}$ Quantification of the $\mathrm{p}$-Erk/Erk and $\mathrm{p}$ AKT/AKT ratio in a and $\mathbf{b}$. e Representative Western blot in mesentery, aorta, and hearts. SD rats that were treated with $50 \mathrm{mg} / \mathrm{kg} \mathrm{MLB}$ for $4 \mathrm{~h}$ were anesthetized, and the mesentery vessels, aorta, and hearts were isolated for the Western blot analyses ( $n=4-6$ per group). $\mathbf{f}$ Quantification of the $\mathrm{p}$-AKT/AKT ratio in e. Data are expressed as the mean $\pm \mathrm{SD}$. ${ }^{*} P<0.05,{ }^{*} P<0.01$ vs. the vehicle group

(Supplementary Fig. S1b) or the protein level (Fig. 5a), as detected by real-time PCR and western blotting analysis, respectively. However, we discovered the increase of eNOS phosphorylation at Ser1177 (p-eNOS) upon treatment with MLB (Fig. 5a). Semiquantification of the level of $p$-eNOS indicated a dose-dependent increase in the $\mathrm{p}$-eNOS/eNOS ratio following MLB treatment (Fig. 5b).

We further confirmed this result in vivo. Changes in the P-eNOS level in the mesentery, aorta, and heart tissues that were obtained from MLB-treated rats were examined as described above. MLB $(50 \mathrm{mg} / \mathrm{kg}$ ) treatment resulted in an apparent increase in the level of p-eNOS level in these tissues, especially in mesentery vessels (Fig. 5c, d). Consistent with the in vitro results, there was no detectable change in the level of eNOS protein. These results suggested a possibility that MLB promoted eNOS enzymatic activity via upregulating phosphorylation at serine 1177 . To further confirm the involvement of stimulated eNOS in MLBrestored blood perfusion in vivo, rats with dextran-induced microcirculation disturbances were pretreated with $L-N A M E$, an eNOS inhibitor, followed by the administration of MLB. We discovered that $L$-NAME could largely reverse the protective effect of MLB on microcirculation dysfunction (Fig. 5f, g). These results confirmed that the activation of eNOS by MLB accounted for the protective effect of MLB on microcirculation disturbance.

MLB increases AKT phosphorylation in vitro and in vivo It remained unclear how eNOS phosphorylation was stimulated by MLB. It was previously reported that the regulation of eNOS activity was highly dependent on the AKT [18] and ERK [19] pathways. We hence examined the impact of MLB on ERK and AKT phosphorylation in HMEC-1 cells. Although ERK phosphorylation was not affected by MLB (Fig. 6a, c), we observed that MLB could 
a

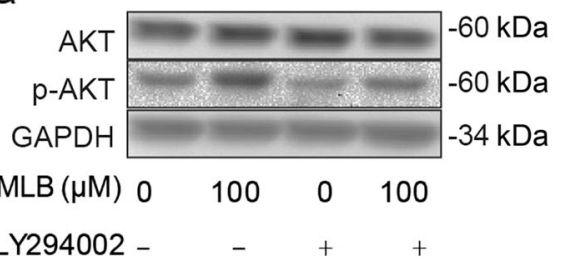

C

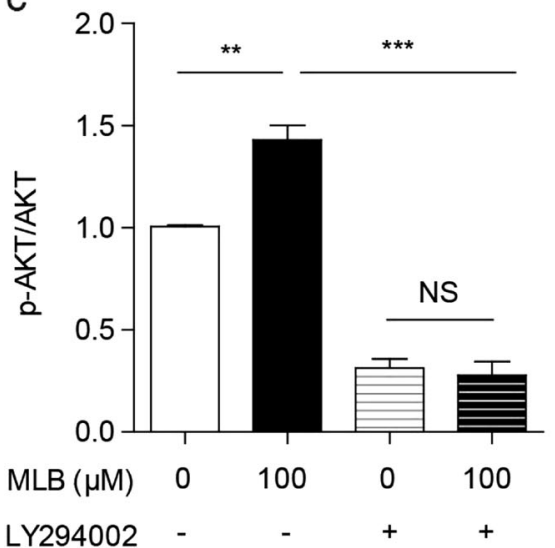

b

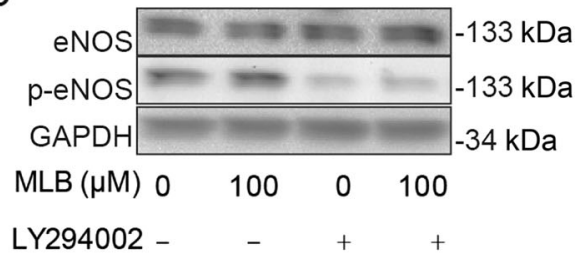

d

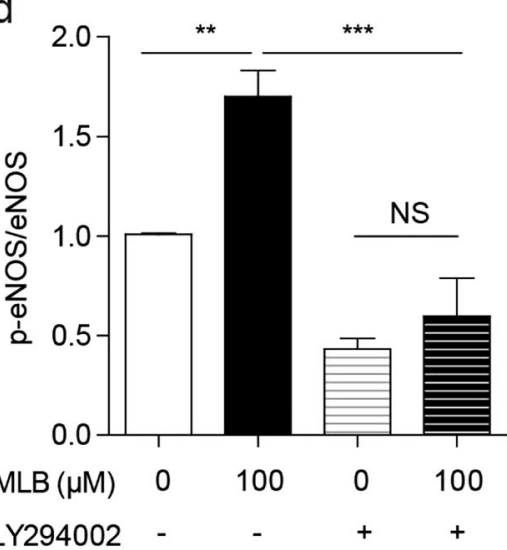

Fig. 7 MLB activated eNOS through the PI3K/AKT pathway. a Western blot analyses of the MLB-induced AKT Ser473 phosphorylation in the presence or absence of the PI3K inhibitor LY294002. LY294002 was added $30 \mathrm{~min}$ before the application of MLB. b Western blot analyses of the MLB-induced eNOS Ser1177 phosphorylation in the presence or absence of PI3K inhibitor LY294002 pretreatment. c and d Quantification of protein expression in $\mathbf{a}$ and $\mathbf{b}$. Data are expressed as the mean \pm SD. ${ }^{*} P<0.05,{ }^{*} P<0.01$

stimulate AKT phosphorylation ( $p-A K T)$ at the serine 473 site in a dose-dependent manner (Fig. 6b, d).

We also examined the p-AKT level in animal vessels. Western blot analyses of the rat mesentery, aorta, and heart showed that the p-AKT level was significantly increased in all of these tissues, with the most potent impact on the mesentery (Fig. 6e, f). This observation was closely associated with the alteration in the p-eNOS level that was caused by MLB treatment, thus supporting a hypothesis that AKT might account for the increase in p-eNOS following MLB treatment.

PI3K inhibitor blocks MLB-enhanced eNOS phosphorylation We finally validated whether AKT signaling was required for the upregulation of p-eNOS that was caused by MLB. To this end, HMEC-1 cells were pretreated with LY294002, a widely used inhibitor targeting phosphoinositide 3-kinase (PI3K), to block downstream AKT phosphorylation. As expected, pretreatment with LY294002 $(10 \mu \mathrm{M})$ largely blocked the enhancement of AKT phosphorylation that was caused by MLB treatment (Fig. 7a, c). Accordingly, the effect of MLB on the activation of eNOS was abolished as well (Fig. 7b, d). These results confirmed that PI3KAKT signaling mediated the activation of eNOS and the resultant NO production in endothelial cells.

\section{DISCUSSION}

Magnesium lithospermate $\mathrm{B}$ is the main constituent of Salvia miltiorrhiza, which has been demonstrated to have various active properties, including elevating the ability of antioxidation, affecting blood lipid metabolism, and inhibiting the generation of atherosclerosis [20]. The pharmacological effects of MLB on cardiovascular disease have also been demonstrated in various experimental models [9], but its molecular mechanisms remain elusive. MLB was shown to inhibit $\mathrm{H}_{2} \mathrm{O}_{2}$-induced myocardial cell apoptosis through the PI3K/AKT pathway [21]. MLB was also shown to reduce the expression of VCAM- 1 and ICAM- 1 to protect endothelial function [22]. Another study confirmed that MLB could promote arterial vasodilation through the activation of $\mathrm{BKCa}$ channel of smooth muscle cells [23]. In the present study, we reported a novel pharmacological effect of MLB that could ameliorate microcirculation dysfunction mainly through endothelial-dependent vasodilation and NO release Fig. 8. These findings provide an experimental basis for expanding the therapeutic benefits of MLB and Salvia phenolic acid.

$\mathrm{NO}$ is a principal mediator in several physiological responses [24]. In 1980, Furchgott demonstrated that the vascular relaxation that was induced by ACh was mediated by a labile humoral factor, later named as endothelium-derived relaxing factor (EDRF). In 1986, Furchgott and Ignarro speculated that EDRF may be NO [25]. NO enhances vascular relaxation and improves diastolic distensibility. Moreover, NO was also shown to inhibit platelet aggregation and adhesion $[26,27]$. In the present study, we showed that MLB significantly enhanced NO production, which may promote microcirculation perfusion via vascular relaxation.

NO is synthesized by the enzyme nitric oxide synthase, and the calcium-dependent constitutive eNOS is shown to be present in the endothelium throughout the body. It has been recognized that decreased eNOS bioactivity occurs very early in atherogenesis. Posttranslational modifications, and phosphorylation in particular, represent a key mechanism for regulating the activity of eNOS [28]. Although eNOS can be phosphorylated on serine, threonine, and tyrosine residues, the most well-known functional consequences of phosphorylation are those of serine $1177 / 1179$ in the reductase domain. The phosphorylation of eNOS at Ser1177 is pivotal in the direct regulation of NO production [18]. We herein discovered that MLB treatment could induce a rapid increase of eNOS phosphorylation at Ser1177 in endothelial cells, and the in vivo study showed that the eNOS inhibitor L-NAME could reverse the protective effect of MLB on microcirculatory disturbance, suggesting the function of MLB in promoting the enzymatic activity of eNOS. Stefanie et al. have demonstrated that the serine/threonine protein kinase AKT/ PKB mediates the activation of eNOS [18], leading to an increase in 


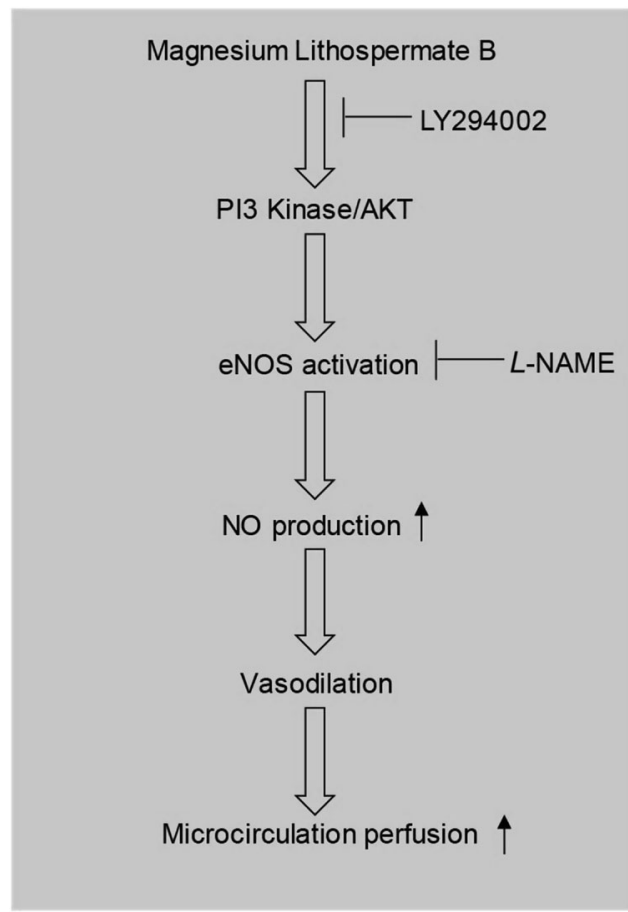

Fig. 8 A scheme showing the impact of MLB on microcirculation perfusion. MLB increases microcirculation perfusion via activating the PI3K/AKT pathway and increasing eNOS phosphorylation, which in turn increases NO production in rat vascular tissues

NO production. Here, we also found that MLB could activate the AKT pathway in endothelial cells and that the PI3K inhibitor LY294002 could abolish the effect of MLB on AKT activation. This evidence provides a molecular link between eNOS activation and MLB treatment. Notably, previous studies have also suggested the activation of PI3K/AKT by MLB, which may contribute to the reduced necrosis and apoptosis in H9c2 cardiomyocytes [29] and its neuroprotective effects on cerebral ischemia/reperfusion injury [30]. However, it remains unclear to us how MLB activates the PI3K-AKT pathway. It is also worth mentioning that we have examined the impact of MLB on the mesentery, aorta, and heart in parallel, and we have observed that MLB can increase eNOS phosphorylation and AKT phosphorylation in these three vascular tissues, but the most potent impact of MLB was observed in the mesenteric vessels. This might be due to the different sensitivity of various vascular beds (Figs. 5, 6).

In the dextran-induced microcirculation dysfunction model, the rat hindlimb perfusion was restored at $10 \mathrm{~min}$ following the MLB injection, and this effect was sustained for up to $80 \mathrm{~min}$ until the experimental end point $(P<0.05)$. This finding shows that the subcutaneous injection of MLB may be advantageous for a more sustained therapeutic effect. In contrast, an intravenous injection of MLB rapidly increased mesenteric microperfusion within less than $1 \mathrm{~min}$, but this effect disappeared immediately, likely due to the rapid elimination of MLB in vivo. To exclude the possibility that this difference resulted from distinct mechanisms in different tissues, we examined the total serum nitrite levels and the eNOS status in the mesenteric vessels $2 \mathrm{~min}$ after an intravenous administration of MLB. MLB similarly appeared to increase the total nitrite levels in rat serum and activated eNOS in rat mesenteric vessels following a subcutaneous injection (data not shown). These results together suggest that the subcutaneous injection of MLB could prolong its pharmacological effects in improving the microcirculation blood flow.

Together, this study revealed that the PI3K/AKT/eNOS pathway might be one of the pathways that is triggered by MLB leading to
NO release and improved microcirculation perfusion. As NO is necessary for vasodilation, vascular remodeling and the decreased bioavailability of NO facilitates the pathophysiological progress in vascular wall morphology. The treatment with MLB, which resulted in increased PI3K/AKT/eNOS-mediated NO production, might provide a solid method for improving microcirculation dysfunction.

\section{ACKNOWLEDGEMENTS}

We thank Prof Yi-ping Wang for the experimental design and financial support and for his considerate supervision and invaluable suggestions to students, which will benefit us for the rest of our lives. His sudden passing grieves us, and his contributions can only be appreciated in the acknowledgements section according to the authorship regulations of ICMJE. We thank Min Huang for the language editing and Jing Zhao, Zi-ying Shen, Wen-wei Xu, Liang Hu, Fei Gao, Cong Xi, Hui-hui Li, and Jiao-meng Li for their technical assistance.

\section{AUTHOR CONTRIBUTIONS}

YLL and XYZ designed the study; LX provided the MLB and supervised this study; $Y L L$ and $X Y Z$ performed the experiments; YLL wrote the manuscript. All of the authors have read, revised. and approved the submitted version of the manuscript.

\section{REFERENCES}

1. Gutterman DD, Chabowski DS, Kadlec AO, Durand MJ, Freed JK, Ait-Aissa K, et al. The human microcirculation: regulation of flow and beyond. Circ Res. 2016;118:157-72.

2. Ellis CG, Jagger J, Sharpe M. The microcirculation as a functional system. Crit Care. 2005;9(Suppl 4):S3-8.

3. Abularrage CJ, Sidawy AN, Aidinian G, Singh N, Weiswasser JM, Arora S. Evaluation of the microcirculation in vascular disease. J Vasc Surg. 2005;42:574-81.

4. Serne EH, de Jongh RT, Eringa EC, IJzerman RG, Stehouwer CD. Microvascular dysfunction: a potential pathophysiological role in the metabolic syndrome. Hypertension. 2007;50:204-11.

5. De Boer MP, Meijer RI, Wijnstok NJ, Jonk AM, Houben AJ, Stehouwer CD, et al Microvascular dysfunction: a potential mechanism in the pathogenesis of obesity-associated insulin resistance and hypertension. Microcirculation. 2012; 19:5-18.

6. Crea F, Camici PG, Bairey Merz CN. Coronary microvascular dysfunction: an update. Eur Heart J. 2014;35:1101-11.

7. Task Force M, Montalescot G, Sechtem U, Achenbach S, Andreotti F, Arden C, et al. 2013 ESC guidelines on the management of stable coronary artery disease: the Task Force on the management of stable coronary artery disease of the European Society of Cardiology. Eur Heart J. 2013;34:2949-3003.

8. Li ZM, Xu SW, Liu PQ. Salvia miltiorrhizaBurge (Danshen): a golden herbal medicine in cardiovascular therapeutics. Acta Pharmacol Sin. 2018;39:802-24.

9. Wang J, Xiong X, Feng B. Cardiovascular effects of salvianolic acid B. Evid Based Complement Alternat Med. 2013;2013:247948.

10. Chen YJ, Lo YH, Chen YT, Lai NW, Lin NH, Chung TY, et al. Magnesium lithospermate B improves metabolic changes in high-fat diet-fed rats with metabolic syndrome. J Funct Foods. 2015;14:163-73.

11. Tzen JT, Jinn TR, Chen YC, Li FY, Cheng FC, Shi LS, et al. Magnesium lithospermate $B$ possesses inhibitory activity on $\mathrm{Na}^{+} \cdot \mathrm{K}^{+}$-ATPase and neuroprotective effects against ischemic stroke. Acta Pharmacol Sin. 2007;28:609-15.

12. Chang $C Z$, Wu SC, Kwan AL. Magnesium lithospermate $B$, an active extract of Salvia miltiorrhiza, mediates $\mathrm{SGC/cGMP/PKG} \mathrm{translocation} \mathrm{in} \mathrm{experimental}$ vasospasm. Biomed Res Int. 2014;2014:272101.

13. Kim SH, Kim SH, Choi M, Lee Y, Kim YO, Ahn DS, et al. Natural therapeutic magnesium lithospermate $B$ potently protects the endothelium from hyperglycaemia-induced dysfunction. Cardiovasc Res. 2010;87:713-22.

14. Wu XJ, Wang YP, Wei W, Sun WK, Xu YM, Xuan LJ. Free radical scavenging and inhibition of lipid peroxidation by magnesium lithospermate B. Acta Pharmacol Sin. 2000;21:855

15. Li XF, Wang YP. Depside salts from Salvia miltiorrhiza improve myocardial microperfusion in rats using laser Doppler flowmetry. Acta Pharmacol Sin. 2007;28:789-95.

16. ZY Zhen, Guo YC, Zhang ZG, Yan L, Ge PJ, Jin HM. Experimental Study on microthrombi and myocardial injuries. Microvasc Res. 1996;51:99-107.

17. Palmer RM, Ferrige AG, Moncada S. Niric oxide release accounts for the biological activity for endothelium-derived relaxing factor. Nature. 1987;327: 524-6. 
18. Fulton D, Gratton JP, McCabe TJ, Fontana J, Fujio Y, Walsh K, et al. Regulation of endothelium derivednitric oxide production by the protein kinase Akt. Nature. 1999;399:597-601.

19. Bernier SG, Haldar S, Michel T. Bradykinin-regulated interactions of the mitogenactivated protein kinase pathway with the endothelial nitric-oxide synthase. J Biol Chem. 2000;275:8.

20. Wu WY, Wang YP. Pharmacological actions and therapeutic applications of Salvia miltiorrhiza depside salt and its active components. Acta Pharmacol Sin. 2012;33:1119-30.

21. Liu CL, Xie LX, Li M, Durairajan SS, Goto S, Huang JD. Salvianolic acid B inhibits hydrogen peroxide-induced endothelial cell apoptosis through regulating $\mathrm{PI} 3 \mathrm{~K} /$ Akt signaling. PLoS ONE. 2007;2:e1321.

22. Chen YH, Lin SJ, Ku HH, Chen YL. Salvianolic acid B attenuates VCAM-1 and ICAM1 expression in TNF-a-treated human aortic endothelial cells. J Cell Biochem. 2001;82:512-21.

23. Zhang HF, Chen XQ, Hu GY, Wang YP. Magnesium lithospermate $B$ dilates mesenteric arteries by activating BKCa currents and contracts arteries by inhibiting Kv currents. Acta Pharmacol Sin. 2010;31:665-70.
24. Clough GF. Role of nitric oxide in the regulation of microvascular perfusion in human skin in vivo. J Physiol. 1999;516.2:549-57.

25. Moncada S, Palmer RM, Higgs EA. Nitric oxide: physiology, pathophysiology, and pharmacology. Pharmcol Rev. 1991;43:109-34.

26. Quyyumi AA, Dakak N, Andrews NP, Husain S, Arora S, Gilligan DM, et al. Nitric oxide activity in the human coronary circulation Impact of risk factors for coronary atherosclerosis. J Clin Invest. 1995;95:1747-55.

27. Kukreja RC, Xi L. eNOS phosphorylation: a pivotal molecular switch in vasodilation and cardioprotection? J Mol Cell Cardiol. 2007;42:280-2.

28. Fleming I. Molecular mechanisms underlying the activation of eNOS. Pflug Arch. 2010;459:793-806.

29. Quan W, Wu B, Bai Y, Zhang X, Yin J, Xi M, et al. Magnesium lithospermate B improves myocardial function and prevents simulated ischemia/reperfusion injury-induced $\mathrm{H} 9 \mathrm{c} 2$ cardiomyocytes apoptosis through Akt-dependent pathway. J Ethnopharmacol. 2014;151:714-21.

30. Cao ZQ, Quan W, Hou SX, Guo C, Ma SB, Zhang W, et al. The natural therapeutic magnesium lithospermate $B$ potently provides neuroprotective effects on cerebral ischemia/reperfusion injury in rats. J Ethnopharmacol. 2015;162:191-8. 